\title{
Prevención primaria de cardiopatía isquémica: la combinación de aspirina con anticoagulación de baja intensidad es más efectiva que cada una por separado
}

Thrombosis prevention trial: randomised trial of low-intensity oral anticoagulation with warfarin and low-dose aspirin in the primary prevention of ischaemic heart disease in men at increased risk.

The Medical Research Council General Practice Research Framework. Meade TW, Wilkes HC, Kelleher CC et al. Lancet 1998;352:233-41.

\section{Objetivo}

Valorar los efectos de la aspirina (A) y la warfarina (W) separadas y en combinación, en la prevención primaria de eventos coronarios mayores.

\section{Diseño}

Ensayo clínico randomizado, factorial* doble ciego, controlado con placebo.

\section{Lugar}

Centros de atención primaria (108), Reino Unido.

\section{Pacientes}

Hombres ( $n=5499$ ) entre 45 y 69 años de alto riesgo coronario.

\section{Intervención}

Inicialmente 1427 hombres fueron asignados a W, hasta alcanzar una Razón Internacional Normatizada (RIN) de 1.5 o Placebo (P). De éstos, 1013 se incorporaron a la fase factorial del estudio recibiendo además $A 75 \mathrm{mg} /$ día, o P. Los restantes 4072 hombres entraron directamente a la fase factorial conformándose los siguientes grupos: $W+A$ $(n=1277), W+P(n=1268), A+P(n=1268), P+P(n=1272)$.

\section{Medición de resultados principales}

Eventos Coronarios (EC): sumatoria de muertes coronarias e Infartos de Miocardio (IAM) fatales y no-fatales. Resultado secundario: Accidentes Cerebrovasculares (ACV).

\section{Resultados principales}

Hubo 410 EC (142 fatales y 268 no fatales). Los tratamientos asignados fueron cumplidos por $2 / 3$ de los pacientes. Los pacientes con W alcanzaron un RIN promedio de 1.47 con una dosis promedio de $4.1 \mathrm{mg} /$ día (de 0.5 a $12.5 \mathrm{mg}$ ) y fueron los únicos que disminuyeron $17 \%(1-30 \%$, $\mathrm{p}=0.04$ ) las muertes por todas las causas (no variaron con A ni WA).

\begin{tabular}{|c|c|c|c|c|}
\hline Tratamiento & RRA & RRR de EC (95\%IC, p) & Explicación de la RRR & $\mathrm{ACV}$ \\
\hline $\begin{array}{l}\text { Warfarina } \\
\text { (WA + WP } \\
\text { vs AP + PP) }\end{array}$ & $\begin{array}{c}2.6 \% \\
(\text { de } 12.4 \\
\text { a } 9.8)\end{array}$ & $\begin{array}{c}21 \% \\
(4-35 \%, p=0.02)\end{array}$ & $\begin{array}{l}\text { Por } \emptyset 39 \% \text { de EC } \\
\text { fatales. } \\
(15-57 \%, p=0.003)\end{array}$ & $\begin{array}{l}\text { ₹ no significativo } \\
\text { de ACV (ni totales } \\
\text { ni subtipos) }\end{array}$ \\
\hline $\begin{array}{l}\text { Aspirina (A) } \\
(W A+A P \\
\text { vs WP + PP) }\end{array}$ & $\begin{array}{c}2.3 \% \\
(\text { de } 11.8 \\
\text { a } 9.5 \text { ) }\end{array}$ & $\begin{array}{c}20 \% \\
(1-35 \%, p=0.04)\end{array}$ & $\begin{array}{l}\text { Por } \emptyset 32 \% \text { de EC no } \\
\text { fatales }(12-48 \% \text {, } \\
p<0.01)\end{array}$ & $\begin{array}{l}\text { \# solo ACV hemo- } \\
\text { rrágico } 0.5 / 1000 \\
\text { personas-año }\end{array}$ \\
\hline$W+A($ vs $P P)$ & $\begin{array}{c}4.5 \% \\
(\text { de } 13.3 \\
\text { a } 8.7)\end{array}$ & $\begin{array}{c}34 \% \\
(11-51 \%, p=0.006)\end{array}$ & $\begin{array}{l}\text { Posible sinergia } \\
\text { de efectos anti- } \\
\text { trombóticos. }\end{array}$ & $\begin{array}{l}\text { ₹ACV hemomá- } \\
\text { gico y fatal (no } \\
\text { totales) y la ruptura } \\
\text { de aneurismas }\end{array}$ \\
\hline
\end{tabular}

RRA: Reducción de Riesgo Absoluto cada 1000 personas-años. RRR: Reducción de Riesgo Relativo*. ø: Disminución.: $\neq$ Aumento

\section{Conclusiones}

La $A$ reduce $E C$ no-fatales y la $W$ todos los $E C$, pero principalmente los fatales. La combinación W+A es más efectiva que cada droga individual en la prevención primaria de EC en hombres de alto riesgo coronario.

\section{COMENTARIO}

Estos resultados, representativos de hombres de alto riesgo coronario de todo el Reino Unido, respaldan la eficacia de la aspirina en prevención primaria de EC (menor que en prevención secundaria), pero únicamente sobre $\mathrm{EC}$ no-fatales y no sobre mortalidad total ni ACV. En los mayores estudios de prevención primaria (médicos americanos y británicos; AAS $325 \mathrm{mg}$ en días alternos y $500 \mathrm{mg} /$ día respectivamente) se reduce combinadamente un 33\% el IAM no-fatal ( $p$ 0.0001) sin diferencias en ACV no-fatal y muertes por ACV o IAM; pero aumentando los ACV discapacitantes $(p=0.016)$. En el presente estudio, el riesgo de ACV hemorrágico atribuible* a la aspirina es sólo 0.5/1000 personas-años y aunque con $75 \mathrm{mg} /$ día de AAS parece ser más segura, debe evaluarse el riesgo-beneficio a la hora de recomendarla. La W reduce todos los EC (principalmente los fatales), disminuyendo además la muerte por todas las causas, sin afectar la incidencia de todos los ACV. En estudios de prevención secundaria, no necesariamente homologables a los de prevención primaria, tanto $\mathrm{W}$ como $\mathrm{A}$ reducen ambos tipos de $\mathrm{EC}$, aunque la $\mathrm{A}$ parece tener menor efecto sobre los fatales. La mayor reducción de EC con tratamiento combinado parece indicar que la antiagregación y la modificación de producción de fibrina asociadas son sinérgicas.

La W+A reduce todos los EC. Aunque aumenta los ACV fatales y hemorrágicos (exceso en la tasa de eventos de 1.5 y $0.9 / 1000$ perso- nas-año respectivamente) previene 12 veces más EC que los ACV que ocasiona. No provoca más hemorragias mayores extracerebrales que la A o W separadamente y ningún grupo más que el $\mathrm{P}$ (aunque el poder para detectar éstas era bajo). El riesgo de sangrados mayores y moderados fue similar al de la A, pero se registró un aumento de incidencia de rupturas de aneurismas aórticos y disecantes, por lo que cabría plantearse la necesidad de una ecografía previa para detectar estos pacientes. Tanto con W como con A, es importante el estrecho control de la tensión arterial para prevenir ACV hemorrágico. El inconveniente de la W es que requiere repetidos ajustes de dosis según el RIN, aunque con la anticoagulación de baja intensidad, una vez estabilizados, podrían hacerse cada 3-6 meses. Si bien su eficacia*, (con rigurosos controles) ha sido demostrada, debemos tener cuidado de no extrapolarlo como efectividad* (aplicación práctica) y también considerar los costos de la estrategia (aparentemente equilibrados en prevención secundaria). Se necesitarían tratar 200 hombres de mediana edad y alto riesgo coronario con W+A o 330 con Wo A por un año para prevenir 1 EC (NNT). Los resultados de este estudio alentarían más el uso de W (con o sin A) que la A individualmente, que sólo reduce EC no-fatales; siempre y cuando el paciente y el médico decidan asumir el riesgo de un serio sangrado (similar al de (a A), que aunque pequeño es real.

\section{Dr. Agustín Ciapponi}

Unidad de Medicina Familiar y Preventiva. Hospital Italiano de Buenos Aires.

*Ver Glosario

\section{Referencias}

1. Canadian Task Force on Periodic health examination, 1991 update: AAS and the primary prevention of cardiovascular disease. CAN MED ASSOC J 1991; $145(9): 1091-5$.

2. Antiplatelet Trialists' Collaboration. Collaborative overview randomised trials of antiplatelet therapy-I: Prevention of death, myocardial infarction, and stroke by prolonged antiplatelet therapy in various categories of patients. BMJ 1994; 308: 81-106.

3. Anticoagulants in the Secondary Prevention of Events in Coronary Thrombosis (ASPECT) Research group. Effect of Long term-oral anticoagulant treatment on mortality and cardiovascular and morbidity after myocardial infarction. Lancet 1994; 343:499-503 